\title{
User Satisfaction of Ali Wangwang, an Instant Messenger Tool
}

\author{
Jie Gao and Zhenghua Zhang \\ UED Taobao.com, Huaxing Road 99, Xihu District, \\ Hangzhou, Zhejiang, P.R. China \\ xuezhi.gj@taobao.com
}

\begin{abstract}
Ali Wangwang is an instant messenger that is used by sellers and buyers to get an agreeable dealing about goods listed on Taobao.com. Buyers from Taobao.com stated that they used Ali Wangwang to communicate with sellers before almost every transaction. The objective of this study was to understand the primary factors that affect the user experience of buyers using Ali Wangwang. We designed self-reported questionnaires with questions focusing on users' overall satisfaction and evaluations of the messenger's interface and function, privacy protection, spam messages control and other related properties. We found that properties related to ease-of-use were the most significant factors in predicting user satisfaction.
\end{abstract}

Keywords: satisfaction score, instant messenger, e-commerce.

\section{Introduction}

According to our records, Taobao.com, founded in 2003 by the Alibaba Group, is the largest online retail platform in China with more than 370 million registered users (at the end of 2010). It provides access for businesses and individuals to open online retail stores that serve buyers across mainland China and beyond. Ali Wangwang is an instant messaging platform that was developed by the Alibaba Group. It is used by sellers and buyers to reach agreements regarding goods on Taobao.com. Based on our previous unpublished survey, approximately $99 \%$ of buyers at Taobao.com reported that they used Ali Wangwang to communicate with sellers at least once before making a purchase. More importantly, over $77 \%$ buyers reported that they used Ali Wangwang to communicate with sellers before every transaction at Taobao.com. Despite the large amount of people using Ali Wangwang, we have not systematically explored important factors that affect user satisfaction with this instant messaging platform. The objective of this study is to determine users' satisfaction with Ali Wangwang. Specifically, we would like to understand the factors that can predict the users' satisfaction scores to Ali Wangwang.

\subsection{Background Information and Hypotheses}

Customer satisfaction is a reflection of the degree of the positive feelings of customers towards a service provider. It is an important indicator of a customer's intention to 
repurchase from that provider [1]. For an online retail platform, we believe that customers' satisfaction with Taobao.com's services is influenced by the user experience of the trading website, the experience of communicating with sellers and other off-line factors such as shipping services. Here, we are only interested in exploring factors that may affect the overall satisfaction of buyers at Taobao.com when they communicate with sellers using Ali Wangwang.

Instant messenger (IM) is a convenient communication technology that is used on the Internet. A previous survey by CNNIC showed that over $70 \%$ of Chinese internet users have used at least one type of IM [2]. Most IMs provide services for friends or family members to communicate and strengthen their relationships. However, the users of Ali Wangwang are mostly buyers and sellers, and most buyers use Ali Wangwang to communicate with sellers about goods and services and to negotiate an acceptable price. Therefore, the content of communications on Ali Wangwang is mostly about goods found on Taobao.com. Previous research has suggested that consumers evaluate the online shopping environment including the online shopping website and the item display webpage [3]. We believe that the smoothness and ease of using Ali Wangwang may have a very important impact on users' overall satisfaction with their online shopping experience at Taobao.com. Our hypothesis is that Ali Wangwang's ease-of-use has a positive effect on user satisfaction scores.

The user satisfaction of general IM or mobile IM tools in China captured researchers' attention [e.g., 4, 5]; however, these results may not fully capture Ali Wangwang's special role in buyers' online shopping experience at Taobao.com. A CNNIC survey showed that when Chinese users utilized IMs, they were not only chatting with friends but also playing online games, watching online TV and performing other activities [6]. It is possible that, for IM users in China, the various entertainment functions of IMs may be as important as the chatting function. However, all previous research was based on a general IM tool; no research has discussed how users evaluate of the chatting (communication) function and non-chatting function (entertainment functions and other extended functions) of an IM in a retail context. It is possible that the chatting function, rather than the entertainment function, is of the primary importance to users' satisfaction with Ali Wangwang, because the goal of buyers with Ali Wangwang is to gain more information about the goods or services. Our hypothesis is that entertainment or other extended functions other than chatting may not influence the satisfaction of Ali Wangwang users.

Researchers have asserted that trust is a very important factor for building and maintaining relationships, and trust is also an important factor for the success of an ecommerce website [7], research showed that trust has a positive influence on a user's satisfaction with an e-commerce website [5]. Particularly, through our personal communications with users, we found that buyers sometimes did not want to share their purchasing history with other people for various reasons including embarrassment or concerns with identity theft. We believe that the protection of privacy and personal purchasing information is a crucial part of building users' trust to Taobao. Although we are aware that privacy and safety protections are very important for gaining users' trust and have put a great deal of effort into privacy and safety protections, we do not know if users have perceived our effort or whether these protections positively affect user satisfaction with Ali Wangwang. We assumed that for those users who perceived our efforts of privacy and safety protections would be more satisfied to Ali Wangwang. 


\section{Research Method}

\subsection{Questionnaire}

Currently, Ali Wangwang has two versions: a buyer version, which is like a regular IM tool, and a seller version, which is more like an online call center. It enables sellers to employ multiple people for customer service through just one Ali Wangwang account. We are interested in user satisfaction with Ali Wangwang as an IM tool; therefore, our study was only concerned with buyers.

We designed a self-reported questionnaire with questions that focused on buyers' overall satisfaction and contained the following five aspects that may be relevant to daily usage of Ali Wangwang: ease-of-use, privacy and safety protections, spam messages, the number of functions used and the number of years of experience with Ali Wangwang.

To determine ease-of-use, we focused on understanding the aspects that affect the daily usage of Ali Wangwang. Ease-of-use was measured by the following six items that evaluated the most common aspects that users experienced in their daily usage: an evaluation of Ali Wangwang's interface, an evaluation of the chatting function, an evaluation for log-in speed and success rate, an evaluation of personal computer performance speed while running Ali Wangwang, and the frequency of unexpected software crashes. Each of these items was measured using a 4-point satisfaction evaluation scale ranging from extremely satisfied (4) to extremely unsatisfied (1).

As this IM tool is directly related to the online retail business, we believed that users would also be concerned with protections of privacy and safety, as well as avoiding spam messages. Spam messages were defined as irrelevant promotional information from sellers and when unknown people asked to join a user's friend list. Privacy and safety protections were also measured by the 4-point satisfaction scale. We measured the amount of spam messages by asking users for the number of spam messages they thought they had received in the past month.

Ali Wangwang provides not only a chatting service but also some convenience in functionality that is relevant to online trading (e.g., screen shots, a calculator, sending short text messages, and remote help), online gaming (e.g., casual games on Taobao.com), online financial services (e.g., paying utility fees and paying credit cards) and others. We wanted to know how many extended functions, not including communication or chatting, were used by our buyers. Therefore, we listed the extended functions and asked users to indicate functions they used other than chatting.

In addition to the services that Ali Wangwang provides, we measured the number of years of experience with Ali Wangwang. Although most users adopt Ali Wangwang for online shopping, there are some who use Ali Wangwang as a work communication tool, or for other personal purposes. Therefore, we asked users to report when they began using Ali Wangwang to communicate with sellers when making purchases in our questionnaire.

\subsection{Participants}

Our questionnaire was conducted using an Ali Wangwang pop-up window for seven days. Each day, we randomly selected 200,000 users and popped out a survey window 
while Ali Wangwang users were online. In total, 42,897 users opened our questionnaire, and 16,746 users answered the questionnaire during the survey period.

\section{Results}

Before any analysis was made, we checked the participants' responses to all of the questions and found that only 15,377 participants had fully completed the questionnaire. Therefore, only their responses were included in our data analysis.

The objective of this study was to understand the relationship between user satisfaction and the services provided by Ali Wangwang. Before examining this relationship, we first conducted a series of t-tests to examine whether satisfied and unsatisfied users would have the same attitude toward the items that we used the in the questionnaire. Our results showed that the satisfied users (who gave a satisfaction score of 3 or 4) gave higher scores to all aspects than the unsatisfied users (who gave a satisfaction score of 1 or 2). Because we had a very large sample size, to avoid any significant statistical differences due to the sample size, we also employed effect size to evaluate the differences between users with high and low satisfaction scores. The t-test results and effect sizes (Cohen's d) are shown in Table 1.

The largest effect size between satisfied and unsatisfied users came from the evaluation of the communication (chatting) function, followed by the number of spam messages users received and privacy and safety protections. The smallest differences were found in years of experience using Ali Wangwang and the number of extended functions used.

Table 1. The t-test results $(\mathrm{df}=15,375)$ and effect sizes for the comparison of each item between satisfied and unsatisfied users

\begin{tabular}{lcc}
\hline \multicolumn{1}{c}{ Items } & $t$ & Cohen's $d$ \\
\hline Evaluation of Ali Wangwang's interface & $24.77^{* * * *}$ & .15 \\
Evaluation of Ali Wangwang's chatting function & $32.51^{* * *}$ & .84 \\
Successful log-in rate & $17.26^{* * *}$ & .43 \\
Log-in speed & $12.83^{* * *}$ & .34 \\
Personal computer performance while running & $20.66^{* * *}$ & .47 \\
Ali Wangwang & & .48 \\
Number of unexpected crashes & $21.37^{* * *}$ & .56 \\
Privacy and safety protection & $18.75^{* * *}$ & .63 \\
Number of spam messages & $18.49^{* * *}$ & .12 \\
Experience using Ali Wangwang & -3.99 & .03 \\
Number of extended functions used & -1.02 & \\
\hline
\end{tabular}

indicates $\mathrm{p}<.001$ 
The average overall satisfaction score for participants was 3.19 (out of 4) with SD $=.62$. We conducted a regression analysis to determine whether all aspects of the ease-of-use, privacy and safety protections, frequency of receiving spam messages, number of non-chatting functions that users tried, and years of experience of using would predict participants' satisfaction scores with Ali Wangwang with an alpha level of .05 for all analysis. The main results of the regression analysis are shown in Table 2. We found that in terms of ease-of-use, Ali Wangwang's interface, the chatting function, successful log-in rate and speed, the performance speed of personal computer while running Ali Wangwang, and the frequency of unexpected software crashes were all successful predictors for users' satisfaction with Ali Wangwang. Privacy and safety protections, the frequency of receiving spam messages, and the number of extended functions that users tried were also significant predictors of satisfaction with Ali Wangwang's services.

We did not find that the number of years of experience with Ali Wangwang was a significant predictor to overall user satisfaction scores, suggesting that although users may have used Ali Wangwang for years, they may be not all satisfied with this IM tool.

Table 2. Regression Analysis of User Satisfaction Scores for all Aspects

\begin{tabular}{lc}
\hline \multicolumn{1}{c}{ Items } & Beta \\
\hline Evaluation of Ali Wangwang's interface & $.28^{* * * *}$ \\
Evaluation of Ali Wangwang's chatting function & $.20^{* * *}$ \\
Successful log-in rate & $.07^{* * *}$ \\
Log-in speed & $.03^{* * *}$ \\
Performance speed of personal computer while running & $.08^{* * *}$ \\
Ali Wangwang & $.05^{* * *}$ \\
Number of unexpected crashes & $.04^{* * *}$ \\
Privacy and safety protections & $.04^{* * *}$ \\
Frequency of receiving spam messages & -.00 \\
Years of Experience of using Ali Wangwang & $.01^{* *}$ \\
\hline
\end{tabular}
indicates $\mathrm{p}<.001, *$ indicates $\mathrm{p}<.05$

\section{Summary and Discussion}

In our study, we examined the relationship between overall satisfaction with Ali Wangwang and users' perception of various aspects that we believed were relevant to user satisfaction.

As a communication tool that helps buyers and sellers reach agreements, the most crucial function of Ali Wangwang is chatting. Unsurprisingly, we found that satisfied users were significantly more satisfied with the chatting function than unsatisfied users. Users' evaluation of Ali Wangwang's chatting function is an important and significant predictor to users' overall satisfaction of this IM tool. We found that satisfied and unsatisfied users generally used the same number of non-chatting functions contained in Ali Wangwang. Although the number of non-chatting functions that 
were tried by users was also a significant predictor of user satisfaction scores, its influence on user satisfaction scores was much smaller. Our results seem to indicate that users view Ali Wangwang as a communication tool, and other functions that are not directly related to the ease of communication, such as playing online games, paying for utility fees, which do not greatly affect user satisfaction. However, we also believe that we should not totally abandon the non-chatting functions for two reasons: 1. Even though non-chatting functions were not heavily weighted by Ali Wangwang buyers, we still found that most buyers tried some of the non-chatting functions. As a product, we definitely need to keep these non-chatting functions to meet the users' calls. 2. The limitation of our current study is that we did not measure the time spent on using the non-chatting functions versus the chatting function. It is possible that users spend very limited amount of time on the non-chatting functions, and thus they cannot recall the scenarios of utilizing these functions. For future studies, we suggest to quantify the differences on usage by asking users the frequency and scenarios using both chatting and non-chatting functions.

As expected, privacy and safety protections were also significant factors affecting user satisfaction. The satisfied users stated that they trusted Ali Wangwang with privacy and safety protections and reported receiving less spam messages than the unsatisfied users. However, we speculated that the prediction power of receiving spam messages might be underestimated. We noticed that when we followed up with some users and asked about their opinions on spam message tolerance. Most of them stated that they understood that sometimes sellers need Ali Wangwang to market their online stores and goods to strangers in the same way that retailers make cold calls. Even though in a many cases, the personal interests between buyers and sellers were different. Chinese handle contradiction with compromise and tolerate it by finding a "middle way" that will not harm either side [8]. Chinese buyers do not want to receive spam messages, but their decision making process is also influenced by Chinese culture, resulting in a lower prediction power of the relationship between spam message tolerance and user satisfaction scores.

We asked users about how many years of experience they had of using Ali Wangwang, assuming that users with more experience would have higher satisfaction scores because of their loyalty to the product [5]. To our surprise, the number of years of experience using Aliwangwang was not predictive of the overall satisfaction scores. One explanation is that users with years of experience may have encountered a less polished version of this IM tool, and the past unpleasant experience may have left a strong first impression, biasing them to dislike the tool. The other possibility is that as a product, Ali Wangwang provides multiple updated versions each year. It is also possible that experienced users were still using an older version of Ali Wanwang and thus they have not experienced any of the improvements to Ali Wangwang's ease-of-use.

This study showed that, in the context of online shopping, customer satisfaction with Ali Wangwang primarily depended on factors related to ease-of-use. Our research is just exploration of how users perceive an IM tool during their online shopping. However, we do realize that user satisfaction to this IM tool is not limited to the usability factors we discussed in this short report. There are many other factors, such as, user experience with online shopping, user experience with sellers' attitude during communication, users' experience and knowledge with computers and so on, need further investigations. 


\section{References}

1. Liao, C., Palvia, P., Chen, J.-L.: Information technology adoption behavior Life cycle: Toward a technology continuance theory (TCT). International Journal of Information Management 29(4), 309-320 (2009)

2. CNNIC. Twentieth statistical survey report on the internet development in China (2007), http: / / www. cnnic.cn

3. Demangeot, C., Broderick, A.J.: Consumer perceptions of online shopping environments: A Gestalt approach. Psychology \& Marketing 27(2), 117-149 (2010)

4. Lu, Y., Zhou, T., Wang, B.: Exploring Chinese users' acceptances of instant messaging using the theory of planned behavior, the technology acceptance model, and the flow theory. Computer in Human Behavior 25, 29-39 (2009)

5. Deng, Z., Lu, Y., Wei, K.K., Zhang, J.: Understanding customer satisfaction and loyalty: Am empirical study of mobile instant messages in China. International Journal of Information Management 30, 289-300 (2010)

6. CNNIC. Twentieth statistical survey report on the internet development in China (2006), http: //www. cnnic.cn

7. Lee, M.K.O., Turban, E.: A trust model for consumer Internet Shopping. International Journal of Electronic Commerce 6(1), 75-91 (2001)

8. Peng, K., Nisbett, R.E.: Culture, dialectics and reasoning about contradiction. American Psychologist 54, 741-754 (1999) 